\title{
Expertengespräch: Heuschnupfen und andere Allergien
}

Warum nehmen Heuschnupfen und Allergien Ihrer Auffassung nach zu?

Ausfeld: Ich denke, das liegt an der Umweltverschmutzung.

von Ammon: Das sehe ich auch so. Ein zweiter Faktor ist die Überforderung des Immunsystems: Wir sind generell nicht mehr so tolerant gegenüber äusseren Einflüssen, sodass wir überschiessend auf ganz normale Reize reagieren.

Schapowal: Die Häufigkeit von Allergien stagniert jetzt bei uns. Aber überall dort, wo der westliche Lebensstil eingeführt wird, steigen Allergien nach wie vor an. Man hat das z.B. bei den Ureinwohnern in Grönland, Kanada oder Papua-Neuguinea gesehen; man beobachtet es zurzeit in zahlreichen Entwicklungsländern. In den westlichen Ländern ist etwa ein Drittel der Bevölkerung von einer Allergie betroffen - in der Schweiz 15\% von Heuschnupfen und je $10 \%$ von Asthma und Neurodermitis.

Welche Allergien sind in Ihrem medizinischen Alltag am häufigsten?

von Ammon: Die Hälfte meiner Klienten sind Kinder, von denen wiederum die Hälfte seit Geburt mit Neurodermitis geplagt ist. Dann kommt die Gruppe der 10- bis 12-Jährigen mit

Warum haben Heuschnupfen und Allergien zugenommen? Der Grund scheint der westliche Lebensstil zu sein: Darauf einigen sich Brigitte Ausfeld, Klaus von Ammon und Andreas Schapowal, die wir als Vertreter der TCM, der klassischen Homöopathie und der Phytotherapie zum Expertengespräch gebeten haben. Fest steht auch, dass die Komplementärmedizin bei Allergien viele gute und wirksame Alternativen zu bieten hat.

Heuschnupfen. Einige Kinder entwickeln in diesem Alter auch ein Asthma, oft als Folge von falsch therapiertem Heuschnupfen.

Ausfeld: Die meisten Patienten kommen mit saisonaler Rhinitis, also Heuschnupfen, seltener mit Asthma. Was eindeutig zunimmt, ist die Baumpollenallergie, aber auch allergische Reaktionen auf Gräser und Kräuter haben in der letzten Zeit etwas zugenommen.

Schapowal: Am häufigsten ist sicher der Heuschnupfen, saisonale allergische Rhinitis und Konjunktivitis (Bindehautentzündung), was ja schon im Januar mit den Hasel- und Erlenpollen beginnen kann. Auch ganzjährige Allergien treffe ich an, vor allem Hausstaub- und Tierhaarallergien - Katzen! -, oder Schimmelpilzallergie. Nahrungsmittelallergien sehe ich ebenfalls. Die sind im Kindesalter etwa acht Mal häufiger als im Erwachsenenalter. Die zweitgrösste Gruppe in meiner Praxis sind die Asthmapatienten. Hautkrankheiten sehe ich als HNO-Arzt seltener.

\section{Was ist Ihr Behandlungsansatz?}

Schapowal: Bevor ich behandle, mache ich immer eine genaue Anamnese, d.h., ich frage nach Allergien in der Familie, nach den Hobbys und nach dem Beruf. Als zweiter Schritt kommt die Allergiediagnostik, am wichtigsten der Hauttest mit den häufigsten Inhalations- und Haustierallergenen, ergänzt bei Bedarf durch Blutuntersuchungen. Wenn das Ergebnis des Hauttests nicht mit der Pollenflugzeit und der Beschwerdezeit des Patienten übereinstimmt, muss ein nasaler Provokationstest erfolgen. Auch bei ganzjährigen Beschwerden braucht es diesen. Konkret: Man sprüht das Allergen in die Nase und misst die Symptome - wie oft muss der Patient niesen, wie intensiv läuft die Nase -, wobei das Volumen des nasalen Flusses genau gemessen wird. Dieser Test eignet sich auch sehr gut für das Asthma bronchiale. Für die Therapie kommen dann Allergenkarenz, Hyposensibilisierung und symptomatische Medikamente infrage. Gegen Heuschnupfen gibt es auch ein Phytotherapeutikum, das Pestwurz-Extrakt Ze 339, das seit 2003 zugelassen und auch für Jugendliche ab 12 Jahren auf dem Markt ist.

Ausfeld: In der TCM gibt es die Diagnose «Heuschnupfen» nicht. Die Diagnostik wird anhand des Zungenund des chinesischen Pulsbefundes erhoben. Auf dieser Basis mache ich Körperakupunktur gemäss TCM, hie und da eine Arzneitherapie mit chinesischen Arzneien und meistens Ohrakupunktur. Weil bei diesem Verfahren die verwendeten Dauernadeln bis 


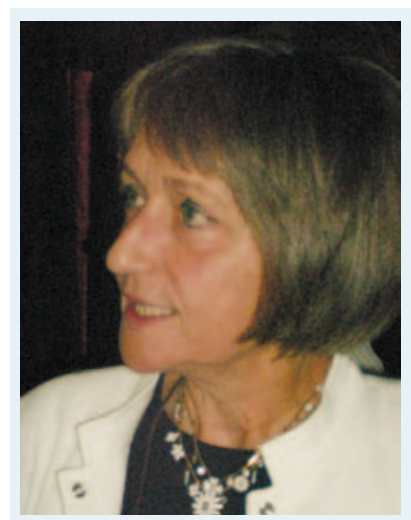

Dr. med. Brigitte Ausfeld-Hafter

(brigitte.ausfeld-hafter@kikom.unibe.ch) ist seit 1995 Dozentin für Traditionelle Chinesische Medizin/Akupunktur an der KIKOM (Kollegiale Instanz für Komplementärmedizin) der Universität Bern. Sie führt seit 1984 eine Praxis für Traditionelle Chinesische Medizin/Akupunktur in Aarau.

«Mein Ansatz ist: So viel schulmedizinische Medikamente wie zur Linderung nötig, so wenig wie möglich wegen der Nebenwirkungen. Dazu kommt die Akupunkturtherapie. Derkombinierte Ansatz klappt häufig.»

zu 10 Tage lang in der Ohrmuschel verbleiben können, hält die Wirkung länger an.

von Ammon: Die homöopathische Theorie geht davon aus, dass sich unterdrückte Hautreaktionen in Schleimhautreaktionen - Nasenlaufen, tränende Augen, Husten - äussern, die, wenn sie wiederum unterdrückt werden, bis auf die Bronchien schlagen und dann weitere sich verschlimmernde Verläufe nehmen können. Das homöopathische Ziel wäre, die Krankheit jeweils auf die weniger bedrohliche Ebene zu verlagern, nach dem Zwiebelschalenprinzip: das Asthma zum Heuschnupfen, den Heuschnupfen zur Hautallergie, diese zu Hautausschlag und -rötung bis zum vollständigen Abheilen. Kurzfristig geht es darum, das Leiden zu lindern. Vor allem bei Kindern ist das wichtig. Das langfristige Ziel ist eine konstitutionelle homöopathische Therapie, d.h. eine Stärkung des Immunsystems und damit der Person.
Was versprechen sich Patienten von Ihrem Ansatz, das sie beim Vertreter der Schulmedizin nicht gefunden haben?

Ausfeld: In den meisten Fällen ist der Grund, die TCM-Praxis aufzusuchen, eine Abneigung gegen schulmedizinische Medikamente. Die Antihistaminika gegen Heuschnupfen sind wirksam, aber sie machen müde und bekämpfen nur die Symptome. Deshalb suchen viele Patienten etwas anderes. Mein Ansatz ist: So viel schulmedizinische Medikamente wie zur Linderung nötig, so wenig wie möglich wegen der Nebenwirkungen. Dazu kommt die Akupunkturtherapie. Der kombinierte Ansatz klappt häufiger. Ich habe $\mathrm{Pa}$ tienten, die seit vielen Jahren kommen, entweder früh in der Saison oder für eine Akuttherapie. Die Erfahrung zeigt, dass sie im Lauf der Zeit nicht mehr jährlich kommen müssen.

von Ammon: Mütter von allergiebetroffenen Kindern wählen oft die Homöopathie, weil sie aufgrund guter Erfahrungen der Methode vertrauen. Erwachsene haben mit schulmedizinischen Methoden oft keinen Erfolg, oder sie leiden unter den Nebenwirkungen. Manchmal haben sie auch die Verschlechterungsspirale erlebt: Hautallergie - Heuschnupfen - Asthma.

Schapowal: Für mich gibt es keinen Widerspruch zwischen der sog. Schulmedizin und der Komplementärmedizin. Die Phytotherapie sehe ich als Teil der traditionellen europäischen Medizin oder Erfahrungsmedizin; das andere wäre die Erkenntnismedizin. Beides ist bestenfalls untermauert durch Good Clinical Practice und Zulassungsverfahren, etwa durch Swissmedic.

Was genau ist der Wirkmechanismus Ihres Ansatzes?

Schapowal: Ich war an den Studien beteiligt, die zur Zulassung des Pest-
wurz-Extraktes Ze 339 geführt haben. Das waren umfangreiche Untersuchungen, angefangen beim Erbgut der Pflanze über die Extraktion bis hin zum Proof of Principle. Die Wirksamkeit des Medikamentes besteht darin, dass bestimmte Inhaltsstoffe der Pestwurz die Biosynthese von Leukotrienen hemmen.

von Ammon: Wir wissen, dass homöopathische Arzneien Auswirkungen auf biologische Systeme haben, seien es Pflanzen, Zellkulturen, Tiere, Kinder oder Erwachsene. Die Erwartungshaltung spielt bei diesen Mechanismen keine Rolle. Das Wirkprinzip heisst similia similibus curentur - «Ähnliches soll durch Ähnliches geheilt werden». Homöopathie wirkt dann am besten, wenn die Krankheitssymptome mit denjenigen Symptomen übereinstimmen, welche die Arzneien bei einem Gesunden hervorrufen.

Ausfeld: Den Wirkmechanismus von TCM für die Diagnose «Atopie» kann man nicht genau beschreiben. "Chinesisch» gesprochen ist es die Lebensenergie Qi, die gestärkt wird. Schlechte Energie wird ausgeleitet.

\section{Gibt es dafür wissenschaftliche Belege?}

Ausfeld: Es gibt immer wieder gute Fallbeschreibungen, aber die rangieren in der Studienhierarchie ganz unten. Insgesamt ist die Studienlage der TCM für Heuschnupfen und andere Allergien eher vage. Ein Kollege an der Charité in Berlin hat mit 400 Probanden vor Kurzem eine vielversprechende Studie begonnen. Doch Akupunktur ist schwierig zu verblinden, weil der Arzt weiss, wohin er sticht, und auch die Sham-Akupunktur - das Stechen an falschen Punkten - hat eine gewisse Wirksamkeit. In der Schweiz ist zudem die Patientenrekrutierung für Studien wegen der benötigten Anzahl von Probanden schwierig, von der Finanzierung ganz zu schweigen. 


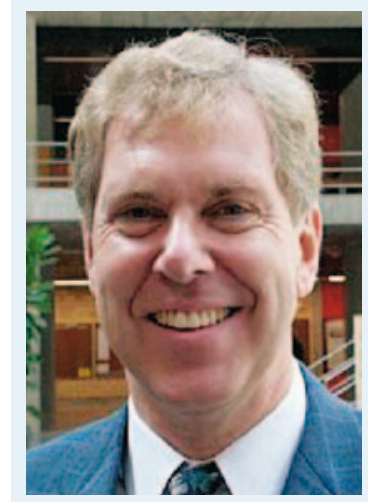

PDDr. med. Dr.h.c. Andreas Schapowal (andreas@schapowal.ch) ist Hals-NasenOhrenarzt und Allergologe/Immunologe mit eigener Praxis in Landquart und Dozententätigkeit an der Medizinischen Hochschule Hannover. Er gilt als ausgewiesener Experte für Phytotherapie.

«Für mich gibt es keinen Widerspruch zwischen der sog. Schulmedizin und der Komplementärmedizin. Die Phytotherapie sehe ich als Teil der traditionellen europäischen Medizin oder Erfahrungsmedizin.»

Schapowal: Wir konnten in randomisierten Doppelblindstudien beweisen, dass das Pestwurz-Extrakt im Vergleich zu zwei bekannten Antihistaminika etwa gleichwertig und einem Placebo überlegen ist. Das Medikament ist verschreibungspflichtig, weil die Pestwurz toxische Stoffe enthält. Die wurden bei Ze 339 aber entfernt; es ist ein sicheres Medikament.

von Ammon: Zum Heuschnupfen gibt es Arbeiten, welche die Wirksamkeit von Homöopathie belegen. Eine neue Studie von Claudia Witt aus Berlin z.B. zeigt, dass Heuschnupfen und Allergien mit Homöopathie mindestens gleich gut behandelbar sind wie mit konventionellen Methoden, dass aber der Aufwand mindestens ebenso gross ist wie bei der Hyposensibilisierung. Ökonomisch ist die konventionelle Medizin kurzfristig gesehen noch im Vorteil. Ich bin aber zuversichtlich, dass, wenn mehr Studiendaten vorliegen, langfristig der Kostenvorteil durch eingesparte Medikamente noch zum Tragen kommt.
Was sind Ihre persönlichen Erfahrungen: Was hilft am besten und warum?

Ausfeld: Bei schwerwiegenden Allergien sollte man früh in der Saison mit Akupunktur beginnen. In der Akutphase ist der Patient mit Ohrakupunktur gemäss Dr. Paul Nogier meist gut behandelt. Ein Patient mit starken Beschwerden muss circa zehn Mal in der Saison kommen; am Anfang im Abstand von 2-3 Wochen, in der Akutphase zweimal wöchentlich. $\mathrm{Ob}$ er jede Saison kommen muss, hängt oft von den kosmischen Bedingungen ab. Wenn es wenig regnet, wüten Allergien besonders heftig. Und dann gibt es auch intrapersonelle Gegebenheiten wie z.B. den Lebensrhythmus. Aber der ist schwer zu bestimmen.

Schapowal: Ich prüfe immer als Erstes, ob man eine ursächliche Behandlung durchführen kann, also die Vermeidung des Allergens; Pollenfilter im Auto und an den Fenstern, den Marathon nicht gerade dann laufen, wenn die Gräser- oder Birkenpollen fliegen. Im Sinne einer Prävention versuche ich, das Verhalten meiner Patienten zu beeinflussen: Wer eine Mehlallergie hat, sollte nicht Bäcker werden, wer Neurodermitis hat, nicht Coiffeuse. Und mit einer Tierhaarallergie sollte man nicht ausgerechnet den Pferdesport ausüben oder Katzen halten. Auf solche Aspekte lege ich grossen Wert. Nummer zwei ist die Hyposensibilisierung. Das empfehle ich oft. Voraussetzung dafür ist, dass der Zusammenhang zwischen Allergenen und Beschwerden eindeutig erwiesen ist. Schliesslich die symptomatische Behandlung mit Medikamenten: Es gibt eine ganze Reihe phytotherapeutischer Medikamente, die etwa für die Behandlung der Haut nützlich sind. Beispielsweise das Nachtkerzenöl, das bereits für Kinder ab 1 Jahr zugelassen ist. Für begleitende Atemwegserkrankungen existieren diverse pflanzliche Arzneien, die entzündungshemmend und schleimlösend wirken, etwa Thy- mian- oder Primel-Extrakte. Wenn jemand mit Heuschnupfen kommt, dann bespreche ich mit dem Patienten, was er möchte. Manche möchten nur Nasenspray oder Augentropfen. Antihistaminika haben den Vorteil, dass sie kostengünstig sind und nur einmal am Tag angewendet werden müssen. Die Symptomlinderung ist bestenfalls 40\%. Damit ist der Patient meistens zufrieden. Pestwurz-Extrakt ist eine gute Alternative, aber nicht besser. Sein grosser Vorteil: Er macht nicht müde. Doch er ist relativ teuer.

\section{Kombinieren Sie Ihren spezifischen Ansatz mit der Schulmedizin?}

von Ammon: Sicherlich, das ist für mich kein Entweder-oder. Ich habe einen massvoll pragmatischen Ansatz, d.h. manchmal muss ich kurzfristig etwas Schulmedizinisches einsetzen, um Ruhe herzustellen. Oberstes Ziel ist, dass das Kind nicht leidet.

Ausfeld: Ja. Da ich grundsätzlich Schulmedizinerin bin, verbinde ich sehr oft beide Therapien. Der Vorteil einer Praxis wie meiner ist der, dass Leute bald bereit sind, Medikamente wegzulassen, wenn sie nicht nötig sind. Alle meine Patienten haben ein Antihistaminikum zu Hause oder, wenn sie zu Asthma neigen, die einschlägigen Asthmamittel. Sie sind zwar kritisch gegenüber Medikamenten, aber bei wirklichem Bedarf setzen sie sie ein. Denn die Akupunktur ist nicht immer zielführend.

\section{Wie beurteilen Sie die sog. \\ Desensibilisierung oder Hyposensibilisierung?}

von Ammon: Zurückhaltend. Wenn das nicht genügend gut vorbereitet ist, kann es erneut zu einer allergischen Reaktion führen. Nicht im klinischen Sinn, sondern vom Erleben her. Für mich kommt eine Desensibilisierung 


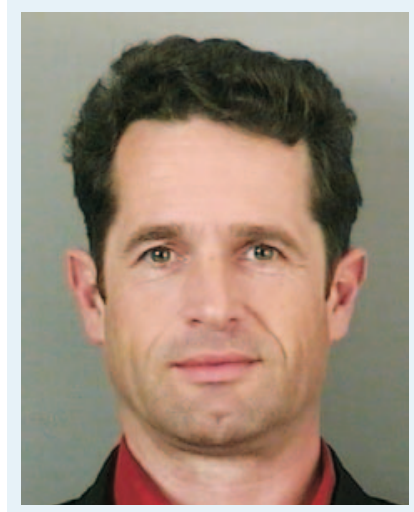

Dr. med. Klaus von Ammon

(klaus@vonammon.ch) ist Oberarzt an der KIKOM (Kollegiale Instanz für Komplementärmedizin) der Universität Bern. Er führt eine eigene Praxis für klassische Homöopathie in Stäfa.

«Zum Heuschnupfen gibtes Arbeiten, welche die Wirksamkeit von Homöopathie belegen. Eine neue Studie aus Berlin zeigt, dass Heuschnupfen und Allergien mit Homöopathie mindestens gleich gut behandelbar sind wie mit konventionellen Methoden.»

erst dann infrage, wenn der homöopathische Ansatz über mehrere Jahre nicht erfolgreich war, und auch dann erst, wenn der Patient für dieses Verfahren homöopathisch gestärkt wurde.

Ausfeld: Persönlich würde ich dies nie machen lassen; der Aufwand ist enorm, und letztlich weiss man wenig über den Nutzen. Meine Patienten konsultieren meine Praxis, weil sie diese Hyposensibilisierung nicht wollen oder weil sie schlechten Erfolg damit hatten. Insofern habe ich eine negative Auslese in meinem Patientengut. Dies verfälscht wohl meinen Eindruck der Wirksamkeit dieses Verfahrens.

Schapowal: Wie gesagt: Ich empfehle sie sehr oft. Es ist die beste Möglichkeit, Inhalationsallergien nachhaltig zu behandeln. Bei Rhinitis, Konjunktivitis und Asthma durch Pollenallergie stehen die Heilungschancen bei etwa $85 \%$, bei Katzenund Hausstaubmilbenallergie bei
$80 \%$, bei den Schimmelpilzen Alternaria und Cladosporium bei immerhin 70\%. Die Lehrmeinung in Bezug auf Neurodermitis ist nicht eindeutig; die wird durch Hyposensibilisierung nicht selten schlechter.

\section{Wirkt Ihr Ansatz auch präventiv?}

Schapowal: Phytotherapeutische Arzneien wirken ausschliesslich symptomatisch. Weder kann man eine Toleranzinduktion noch eine Hyposensibilisierung oder eine dauerhafte Heilung bewirken.

von Ammon: Für zwei Drittel Erfolgschance können wir immer einstehen; in erfahrenen Händen beträgt die Rate $75-80 \%$. Für mich zählt erst ein Ergebnis nach zwei, drei Jahren. Das ist auch eine Frage der Geduld und der Bereitschaft, biologische Prozesse verstehen zu wollen.

Ausfeld: Bei Kindern kann man vor der Saison mit Laserakupunktur gut vorbeugen. Erwachsenen verschreibe ich als Vorbehandlung oft ein homöopathisches Mittel, das ich dann mit Akupunktur kombiniere.

\section{Sollte zu Heuschnupfen und \\ Allergien in Ihrem Bereich \\ mehr geforscht werden?}

Ausfeld: Über kurz oder lang wird auch in der Schweiz die Akupunktur wohl nur für wirkungsevidenten Nutzen von den Krankenkassen bezahlt werden. Das Problem ist, dass es in der chinesischen Medizin keine schulmedizinischen Diagnosen gibt. In Studien werden die Probanden mit den schulmedizinischen Diagnosen mit einer Rezeptakupunktur behandelt, und das entspricht nicht der chinesischen Idee der individuellen Behandlung. In der Schweiz ist es schwierig, gute Forschung zu betreiben. Mein Anliegen ist, dass wir uns international noch besser vernetzen und multizentrische Studien machen können. Die Therapie von Heuschnupfen und Allergien als mögliche Indikation zu erforschen, ist sicher sinnvoll. Die jahrelange Erfahrung in der Praxis zeigt nämlich, dass TCM eine wirksame Therapie ist, relativ kostengünstig und nicht mit so vielen Nebenwirkungen behaftet wie die Schulmedizin.

Schapowal: Forschungsbedarf gibt es z.B. im Bereich der homöopathischen Medikamente. Es wurden sehr interessante Studien gemacht, die zweifelsfrei eine Wirksamkeit vor allem bei der allergischen Konjunktivitis gezeigt haben. Da ist die grosse Frage: Wie wirkt das pharmazeutisch überhaupt? Die Wirksamkeit ist da, aber das Wirkungsprinzip, z.B. von Galphimia glauca, ist unklar. Leider liegt es nicht im Interesse grosser Pharmafirmen, solche Studien im grossen Stil $\mathrm{zu}$ finanzieren, selbst wenn es pionierhafte Forschungsarbeiten in die besten Journals wie Lancet geschafft haben. Beim Pestwurz-Extrakt hätte ich mir gewünscht, dass man weiter geforscht und das Medikament optimiert hätte, etwa in eine retardierte Form, die 12 Stunden wirkt.

von Ammon: Aufgrund der Gleichförmigkeit allergischer Erscheinungen bietet gerade dieses Krankheitsbild einen guten Ansatz für homöopathische Forschung. Es hat einerseits die Ebene der Monomorphie, des Gleichartigen, und darüber hinaus die Ebene der individuellen Ausprägung. Insofern könnte es ein gutes Modell sein. Ich selbst würde mich eher an Hauterscheinungen halten wollen, denn die sind beobachtbar, messbar und wissenschaftlich kommentierbar. Bei Heuschnupfensymptomen - wie viel Milliliter Schleim pro Stunde? - halte ich dies für schwieriger.

Interview: Irène Dietschi 